CERN-TH.6496

May 1992

\title{
Amplitude estimates for multi-Higgs production at high energies
}

\author{
E.N. Argyres*, Ronald H.P. Kleiss and \\ Costas G. Papadopoulos \\ TH Division, CERN, Geneva
}

\begin{abstract}
We present a number of exact and asymptotic results concerning the perturbative tree-level amplitudes for the decay of an off-shell scalar into a large number of onshell scalars. These results are useful in the discussion of whether or not perturbative unitarity will be violated at sufficiently high energies and/or multiplicities.
\end{abstract}

CERN-TH.6496/92

May 1992

* On leave of absence from the Institute of Nuclear Physics, NRCPS ' $\Delta \eta \mu o ́ k \varrho \iota \tau o \varsigma$ ', GR-153 10 Athens, Greece. 



\section{Introduction}

Recently, the question of the convergence of perturbative quantum field theory has come under renewed study. This has to a large extent been motivated by the possibility of instanton-induced $B+L$-violating cross sections at future hadron colliders. In [1] it was argued that such cross sections can be very large (indeed, in the dilute instanton gas approximation they violate the unitarity bound at sufficiently high energies). In such events a large number of weak bosons (W's, Z's, and Higgses) concomitant with the appropriate prompt fermions would be expected. Moreover, the $B+L$-conserving cross section would also be large, roughly equal to the $B+L$ violating one.

Although this issue is by no means settled yet, it suggests the relevance of an investigation into the perturbative cross section for multiboson production: if the nonperturbative cross section is large, it would be somehow surprising if the perturbative one were unobservably small. The perturbative cross section therefore could serve both as a suggestion about the behaviour of the nonperturbative one, and as a possible background should these spectacular effects be actually observed (it is widely agreed that evidencing $B+L$ violation by direct counting of the final-state baryon number is not feasible, and one would have to rely on the high weak-boson multiplicity as a signal).

As regards the convergence of perturbation theory, the issue is most succinctly put by Voloshin [2]: if cross sections are an asymptotic series in the coupling constant rather than a convergent series, this need not worry us overmuch if the quantity under study has low-order contributions; but it becomes problematic when the Born contribution itself is of very high order, as is the case in multiboson production. It is important to know whether or not such cross sections violate unitarity at the Born level.

Several authors $[2,3]$ have attempted to estimate the cross section for the production of a large number of Higgs bosons (this is the simplest of the processes in question since no polarization degrees of freedom come into play). These estimates are questionable since they hold for kinematical configurations that are outside the physical phase-space (all Higgs bosons having both zero momentum and zero energy), or at best at its very edge (all Higgs bosons with momentum zero). Strictly speaking, then, the corresponding cross sections are zero. A mop-up attempt such as using the extreme-relativistic phase-space volume, as in [3], cannot possibly be justified in our view. We hold that reliable estimates of the cross section should use upper or lower bounds on the amplitude that reside inside the physical phase-space. The present paper purports to be a step in that direction.

The layout of the paper is as follows. In section 2 we present analytical results for the number of Feynman diagrams involved in the decay of an off-shell scalar into $n$ on-shell scalars, for various forms of the scalar self-interaction Lagrangian. 
It is frequently claimed that a superexponential increase of this number indicates tree-level unitarity violation. In section 3 we treat the zero-momentum, zero-energy case mentioned above. In section 4 we touch upon the physical phase-space, where all Higgses have zero momentum and energy $m_{H}$ (the Higgs mass). In section 5 we present estimates for the case where the momenta may be non-zero. These last results should form the basis of realistic (upper or lower) bounds on the cross section; they may be used for the problem of tree-level unitarity violation, which we shall address in a forthcoming paper [4]. In all cases we shall be particularly interested in the behaviour of the various amplitudes for large values of the multiplicity.

\section{Diagram counting}

In this section we derive expressions for the number of tree-level Feynman graphs that enter in the decay of an off-shell Higgs into a number $n$ of on-shell Higgses: in these diagrams, only Higgs self-interactions come into play so that the diagram structure is rather simple. We denote by $a(n)$ the number of topologically distinct diagrams. We shall study various self-interaction Lagrangians, using the recursionrelation technique that was introduced in this context in [2].

\subsection{Pure $\phi^{m}$ theory}

If the only self-interaction vertex has $m$ legs, an off-shell scalar can only decay by way of going into $p=m-1$ (internal or external) scalars. Taking into account the various combinatorial factors, we find the following recursion relation for $a(n)$ :

$$
a(n)=\frac{n !}{p !} \sum_{n_{1,2, \ldots, p} \geq 1} \frac{a\left(n_{1}\right)}{n_{1} !} \frac{a\left(n_{2}\right)}{n_{2} !} \cdots \frac{a\left(n_{p}\right)}{n_{p} !} \delta_{n_{1}+n_{2}+\cdots n_{p}, n}
$$

for $n>1$, and of course $a(1)=1$. We make the obvious Ansatz

$$
a(n)=n ! b(n)
$$

which leads to the following equation for the $b(n)$ :

$$
b(n)=\frac{1}{p !} \sum_{n_{1,2, \ldots, p} \geq 1} b\left(n_{1}\right) b\left(n_{2}\right) \cdots b\left(n_{p}\right) \delta_{n_{1}+n_{2}+\cdots n_{p}, n},
$$

with $b(1)=1$. The solution to this recursion was found some time ago by Fuss [5]. The simplest approach is to introduce a generating function $y=y(x)$, as follows:

$$
y(x)=\sum_{n \geq 1} b(n) x^{n},
$$


so that

$$
y=x+y^{p} / p !
$$

The explicit form of $y(x)$ is in this case given by the Lagrange expansion $[6,7]$ :

$$
\begin{aligned}
y & =x+\sum_{k \geq 1} \frac{1}{k !} \frac{d^{k-1}}{d x^{k-1}}\left(\frac{x^{p}}{p !}\right)^{k} \\
& =\sum_{k \geq 0}\left(\frac{1}{p !}\right)^{k} \frac{(p k) !}{k !(q k+1) !} x^{q k+1},
\end{aligned}
$$

with $q=p-1$. We see that, as they should, the $a(n)$ are only non-zero if $n$ is of the form $q k+1$, and for those $n$ we have

$$
\begin{aligned}
a(n) & =(p !)^{-k} \frac{(p k) !}{k !} \\
& \sim \frac{n !}{n^{3 / 2}}\left(\frac{p^{p}}{p ! q^{q}}\right)^{(n-1) / q} \sqrt{\frac{p}{2 \pi}},
\end{aligned}
$$

where the last line is the asymptotic form for large $n$, obtained by the Stirling approximation.

\subsection{Asymptotics from the generating function}

The asymptotic form for $a(n)$ above could be derived straightforwardly since the exact closed form of $b(n)$ is known for a pure $\phi^{m}$ theory. As we shall see, for theories with more possible vertices a closed form does not appear feasible, but we can at least get the asymptotic behaviour directly from the generating function, as follows. Let the generating function for a series $c(n)$ be given as $y=\sum_{n>0} c(n) x^{n}$. A simple recursion relation for the $c(n)$ like the one above for the $b(n)$ will then boil down to an algebraic relation between $x$ and $y$ :

$$
F(x, y)=0,
$$

with $F$ a known function. For $x$ sufficiently close to zero, this equation has necessarily a root, which starts at $c(0)$ at $x=0$ and moves away from it as $x$ departs from 0 . There may be other roots but they do not concern us as long as they are distinct from the first one. The situation changes when $x$ reaches a value for which two roots (the 'physical' one and an 'unphysical' one) coincide: at that point a branch develops in the complex $y$ plane, and the analyticity of the function $y(x)$ breaks down. This is seen to happen at the smallest (absolute) value for $x_{0}$ and the corresponding value $y_{0}$ for which we have, in addition to Eq. (8), also

$$
F_{y}\left(x_{0}, y_{0}\right)=0
$$


where a subscript denotes a partial derivative. It is this point in the $(x, y)$ plane that defines the convergence radius of $y(x)$ and, by Cauchy's theorem [7], the asymptotic form of the coefficients $c(n)$. We may expand $F(x, y)$ around $\left(x_{0}, y_{0}\right)$, taking care to stay on the good side of the convergence limit for the $x$ values:

$$
\begin{aligned}
0= & F(x, y) \\
= & F\left(x_{0}, y_{0}\right)+\left(y-y_{0}\right) F_{y}\left(x_{0}, y_{0}\right)-\left(x_{0}-x\right) F_{x}\left(x_{0}, y_{0}\right) \\
& +\left(y-y_{0}\right)^{2} F_{y y}\left(x_{0}, y_{0}\right) / 2+\cdots ;
\end{aligned}
$$

since, by construction, the first two terms vanish, we have, to lowest order in the deviations from $\left(x_{0}, y_{0}\right)$ :

$$
y \sim y_{0}-\sqrt{\frac{2 F_{x}\left(x_{0}, y_{0}\right)}{F_{y y}\left(x_{0}, y_{0}\right)}}\left(x_{0}-x\right)^{1 / 2} .
$$

Here, the minus sign must be chosen to have $d y(x) / d x>0$ (this holds in cases like the ones we study, where all $c(n)$ must be positive). We have of course assumed that $F_{x}$ and $F_{y y}$ do not vanish at $\left(x_{0}, y_{0}\right)$ : for our problem this always holds. The asymptotic form of $c(n)$ is (to leading order in $1 / n$ ) given by the high-order terms in the expansion of the square root:

$$
\begin{aligned}
\left(x_{0}-x\right)^{1 / 2} & =\sum_{k \geq 0}\left(\begin{array}{c}
1 / 2 \\
k
\end{array}\right)(-1)^{k} x_{0}^{1 / 2-k} x^{k} \\
& =\sum_{k \geq 0}\left(\begin{array}{c}
k-3 / 2 \\
k
\end{array}\right) x_{0}^{1 / 2-k} x^{k} \\
& =-\sum_{k \geq 0} \frac{(k-3 / 2) !}{2 \sqrt{\pi}(k) !} x_{0}^{1 / 2-k} x^{k} .
\end{aligned}
$$

The asymptotic form of $c(n)$ therefore reads

$$
c(n) \sim \frac{1}{x_{0}^{n} n^{3 / 2}} \sqrt{\frac{x_{0} F_{x}\left(x_{0}, y_{0}\right)}{2 \pi F_{y y}\left(x_{0}, y_{0}\right)}} .
$$

The $n^{-3 / 2}$ behaviour is seen to be universal in the sense that it depends only on the locally parabolic shape of $F(x, y)$, as a function of $y$, around the point $\left(x_{0}, y_{0}\right)$. In fact, also subleading terms can be obtained by taking into account the higher derivatives of $F(x, y)$ : for instance the first subleading term, of order $\mathcal{O}\left(n^{-5 / 2}\right)$, comes from the subleading terms in the Stirling approximation of $(k-3 / 2) ! / k !$ as well as from the leading terms of the part of $y$ that goes as $\left(x_{0}-x\right)^{3 / 2}$.

When we want to apply this to our pure- $\phi^{m}$ recursion, a subtlety unfortunately crops up immediately (as we shall see, the mixed case with more vertices is better 
behaved): since $b(n)$ is zero except for $n=q k+1$, it has strictly speaking no asymptotic form if $q>1$. We therefore cast the recursion in a different form by defining a $c(k)$ as

$$
c(k) \equiv b(q k+1)
$$

upon which the recursion relation (1) becomes

$$
c(k+1)=\frac{1}{p !} \sum_{k_{1}, \ldots, p \geq 0} c\left(k_{1}\right) \cdots c\left(k_{p}\right) \delta_{k_{1}+\cdots k_{p}, k},
$$

with $c(0)=1$. With $y=\sum_{k \geq 0} c(k) x^{k}$ we then have

$$
F(x, y)=\frac{x y^{p}}{p !}-y+1
$$

and following the above reasoning we find

$$
y_{0}=\frac{p}{q} \quad, \quad x_{0}=\frac{p ! q^{q}}{p^{p}},
$$

and the asymptotic form we find is precisely that of Eq. (7). The exponential factor $x_{0}^{-k}$, where $x_{0}=8 / 9$ for $\phi^{4}$ theory, was mentioned in [2], and had previously been derived by completely different means in [3]. It is here seen to follow immediately from the recursion relation for the diagram counting.

\subsection{Theories with mixed interactions}

We now turn to the case where vertices with different $m$ are available. This is relevant to the Minimal Standard Model, where both $\phi^{4}$ and $\phi^{3}$ vertices occur, and, inasmuch as we only consider the number of graphs, for purely gluonic QCD as well. In this case, the recursion relation for $a(n)$ is depicted in Figure 1 and reads

$$
a(n)=\frac{n !}{6} \sum_{n_{1,2,3} \geq 1} \frac{a\left(n_{1}\right)}{n_{1} !} \frac{a\left(n_{2}\right)}{n_{2} !} \frac{a\left(n_{3}\right)}{n_{3} !} \delta_{n_{1}+n_{2}+n_{3}, n}+\frac{n !}{2} \sum_{n_{1,2} \geq 1} \frac{a\left(n_{1}\right)}{n_{1} !} \frac{a\left(n_{2}\right)}{n_{2} !} \delta_{n_{1}+n_{2}, n} .
$$

Again putting $a(n)=n ! b(n)$ and $y(x)=\sum_{n \geq 1} b(n) x^{n}$ we now find the following relation:

$$
F(x, y)=y^{3} / 6+y^{2} / 2-y+x=0 .
$$

Although the answer for $y$ in terms of $x$ can again be written down with the Lagrange expansion, the answer is a rather ugly-looking sum of powers of $\left(x^{3} / 6+x^{2} / 2\right)$ and a closed form for the coefficient of $x^{n}$ for large $n$ is not readily derived. Instead, we use the asymptotic method. For $\left(x_{0}, y_{0}\right)$ we now find two solutions, $(\sqrt{3}-4 / 3, \sqrt{3}-1)$ 


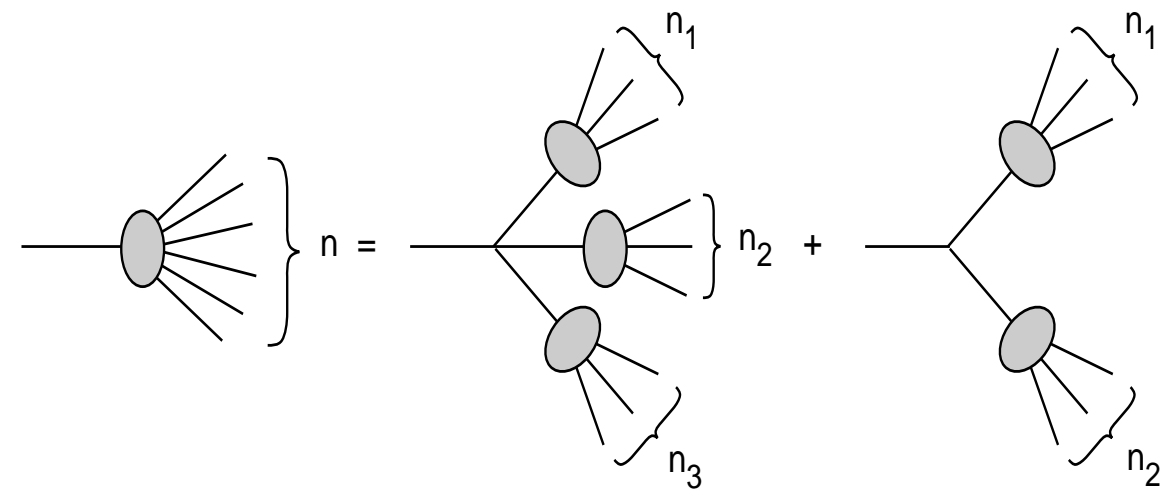

Figure 1: Diagrammatic structure of the recursion relation for mixed $\phi^{4}$ and $\phi^{3}$ interactions.

and $(-\sqrt{3}-4 / 3,-\sqrt{3}-1)$, of which we have to take the first one since it has the smallest value of $\left|x_{0}\right|$. The resulting asymptotic form of the $a(n)$ is therefore

$$
a(n) \sim \frac{n !}{n^{3 / 2}}\left(\frac{9 \sqrt{3}+12}{11}\right)^{n} \sqrt{\frac{3 \sqrt{3}-4}{6 \pi \sqrt{3}}} .
$$

The extension to more complicated (but physically less relevant) mixtures of different self-interactions is straightforward. An interesting case is that of linearized gravity, where vertices of arbitrarily high order enter. It is easily seen that then

$$
\begin{aligned}
F(x, y) & =x-y+\frac{y^{2}}{2}+\frac{y^{3}}{6}+\frac{y^{4}}{24}+\cdots \\
& =e^{y}-2 y-1+x,
\end{aligned}
$$

and the asymptotic form of $a(n)$ is immediately found to be

$$
a(n)=\frac{n !}{n^{3 / 2}}\left(\frac{1}{2 \log 2-1}\right)^{n} \sqrt{\frac{1}{2 \pi}} .
$$

It should be noted that, partly, these results are not new: in Ref. [8] the $n$-dependent part was established, but only after a considerable technical struggle, and the constant term was not found at all.

\section{Amplitudes for zero energy and momentum}

In this section we derive results for the unphysical situation where all Higgses have zero four-momentum. In that case all the internal Higgs propagators have denominator $-m_{H}^{2}$, and the treatment is much like that of the diagram counting. Now, we 
denote by $a(n)$ not the number of diagrams but the actual amplitude. We have, for the pure $\phi^{m}$ theory:

$$
a(n)=\frac{-i \lambda_{m}}{\left(-m_{H}^{2}\right)^{p}} \frac{n !}{p !} \sum_{n_{1,2, \ldots, p} \geq 1} \frac{i a\left(n_{1}\right)}{n_{1} !} \frac{i a\left(n_{2}\right)}{n_{2} !} \cdots \frac{i a\left(n_{p}\right)}{n_{p} !} \delta_{n_{1}+n_{2}+\cdots n_{p}, n},
$$

where we have assumed the Feynman rule $-i \lambda_{m}$ for the $m$-point vertex. Note that we have to take $a(1)=i m_{H}^{2}$ as a starting value for the recursion: when inserting the $a(n)$ in an actual matrix element we have to multiply with the propagator, which reads $-i m_{H}^{-2}$. The solution is trivial since under the Ansatz

$$
a(n)=i n ! m_{H}^{2} b(n)\left(\frac{\lambda_{m}}{-m_{H}^{2}}\right)^{(n-1) / q}
$$

the recursion relation for the $b(n)$ is again that of $\mathrm{Eq}$. (3) with $b(1)=1$, and has the same solution; asymptotically:

$$
a(n) \sim i \frac{n ! m_{H}^{2}}{n^{3 / 2}}\left(\frac{p^{p} \lambda_{m}}{-m_{H}^{2} p ! q^{q}}\right)^{(n-1) / q} \sqrt{\frac{p}{2 \pi}},
$$

for those $n$ that are of the form $q k+1$. Note that, for $\phi^{4}$ theory, we reproduce the exponential factor $p^{p} / q^{q}=27 / 4$ reported in $[2,9]$ and provide a proof that the constant factor is indeed $\sqrt{3 / 2 \pi}=0.691 \cdots{ }^{1}$. The same result was obtained in Ref.[10]

The mixed case of $\phi^{4}$ and $\phi^{3}$ interactions is more involved. Note that in this situation different diagrams may interfere destructively, and no rigorous bound on the amplitude can be derived. On dimensional grounds the two coupling constants are related by

$$
\lambda_{3}=\mu m_{H} \sqrt{\lambda_{4}},
$$

which may be taken as the definition of $\mu$. In spontaneously broken theories like the Higgs sector of the Minimal Standard Model, $\mu$ equals $\sqrt{3}$. Using the Ansatz

$$
a(n)=i n ! m_{H}^{2} b(n)\left(\frac{\lambda_{3}}{-m_{H}^{2} \mu}\right)^{n-1}
$$

we find for the $b(n)$, with $b(1)=1$ :

$$
b(n)=-\frac{1}{6} \sum_{m_{1,2,3} \geq 1} b\left(n_{1}\right) b\left(n_{2}\right) b\left(n_{3}\right) \delta_{n_{1}+n_{2}+n_{3}, n}+\frac{\mu}{2} \sum_{m_{1,2} \geq 1} b\left(n_{1}\right) b\left(n_{2}\right) \delta_{n_{1}+n_{2}, n},
$$

\footnotetext{
${ }^{1}$ In Ref.[2], the coupling constant $\lambda$ is $\lambda_{4} / 6$ in our notation.
} 
so that the equation for the generating function $y=y(x)$ becomes

$$
F(x, y)=-\frac{y^{3}}{6}+\mu \frac{y^{2}}{2}-y+x=0
$$

The convergence limit point $x_{0}$ is now located off the real axis: ultimately this can be traced back to the fact that our kinematical configuration is outside the physical phase-space. Of the two solutions for $\left(x_{0}, y_{0}\right)$ we have to take

$$
\begin{aligned}
x_{0}(\mu) & =\frac{1}{3}\left(\left(\mu^{2}-2\right)^{3 / 2}+3 \mu-\mu^{3}\right) \\
& =1 / 3 \text { for } \mu=\sqrt{3} \\
y_{0}(\mu) & =\mu-\left(\mu^{2}-2\right)^{1 / 2}
\end{aligned}
$$

and we find the asymptotic form for $a(n)$ to be

$$
a(n) \sim i m_{H}^{2} \frac{n !}{n^{3 / 2} x_{0}^{n}}\left(\frac{\lambda_{3}}{-m_{H}^{2} \mu}\right)^{n-1} \sqrt{\frac{x_{0}}{2 \pi \sqrt{\mu^{2}-2}}} .
$$

Note that the value $\mu=\sqrt{3}$ has a somewhat special character since for that $\mu$ the two solutions for $x_{0}$ have the same absolute value. A more striking instance of this will be seen in the next section. Since, as we have already argued, the zero-energy, zeromomentum configuration is not to be used as an estimate of a physical amplitude, we shall pursue this situation no further.

\section{The kinematical threshold}

A more physically relevant estimate for the amplitude can only be obtained by looking at kinematically allowed momentum configurations. For a generic such configuration, the actual recursion relation for the amplitude $a(n)$ is of course horrendously more complicated than we have seen so far, since all possible combinations of invariant masses (that enter in the internal Higgs propagators) come into play. One therefore feels compelled to settle for some simplifying approximation.

One such simplification is to assume that all invariant masses of $k$ Higgs momenta are always as small as they can possibly be, namely $k m_{H}$, and the total $q^{2}$ flowing into a given $a(n)$ is $q^{2}=k^{2} m_{H}^{2}$. This situation was studied in [2] and [3]. Clearly, it can only hold if all Higgses are at rest in the centre-of-mass frame: $\sqrt{s}=n m_{H}$, and in that case the phase-space volume vanishes. At energies above the threshold, the phase space volume is finite but then there must be at least a few invariant masses larger than the minimum. Nevertheless, this zero-momentum (non-zero energy) approximation can always serve as an upper bound on the cross section; Goldberg's 
claim [3] to establish rather a lower bound ${ }^{2}$ is in our view incorrect. In addition, for kinematically allowed momentum configurations, all the diagrams for the decay of a single scalar into scalars will add coherently.

\subsection{Pure $\phi^{m}$ theory}

We shall again start with the pure theory, since this illustrates most simply how we arrive at an exact solution for the amplitude in the zero-momentum case. The recursion formula for the amplitude $a(n)$ is given by

$$
a(n)=-i \lambda_{m} \frac{n !}{m_{H}^{2 p} p !} \sum_{n_{1}, \ldots, p \geq 1} \frac{i a\left(n_{1}\right)}{n_{1} !\left(n_{1}^{2}-1\right)} \cdots \frac{i a\left(n_{p}\right)}{n_{p} !\left(n_{p}^{2}-1\right)} \delta_{n_{1}+\cdots n_{p}, n}
$$

for $n>1$, and under the Ansatz

$$
a(n)=-i m_{H}^{2} n !\left(n^{2}-1\right) b(n)\left(\frac{\lambda_{m}}{m_{H}^{2}}\right)^{(n-1) / q},
$$

we find for the $b(n)$ in this case:

$$
\left(n^{2}-1\right) b(n)=\frac{1}{p !} \sum_{n_{1}, \ldots, p \geq 1} b\left(n_{1}\right) \cdots b\left(n_{p}\right) \delta_{n_{1}+\cdots n_{p}, n},
$$

for $n>1$. The starting value is again $b(1)=1$. Note that, strictly speaking, we have $a(1) \propto 1^{2}-1=0$ but this need not worry us since, as mentioned before, when computing an actual matrix element we would again have to divide by this factor. From inspection of $a(2)$ and $a(3)$ it is clear that $b(1)=1$ is indeed correct.

Owing to the occurrence of the $n^{2}$, the equation for the generating function $y(x)$ is no longer simply algebraic but involves derivatives:

$$
x^{2} \frac{d^{2}}{d x^{2}} y(x)+x \frac{d}{d x} y(x)-y(x)-\frac{1}{p !} y(x)^{p}=0,
$$

with initial conditions $y(0)=0, y^{\prime}(0)=0$. It happens that we can solve this differential equation in a straightforward manner by separation of variables. The result is

$$
y(x)=\frac{x}{\left(1-x^{q} / 2 m !\right)^{2 / q}},
$$

from which $b(n)$ is readily extracted:

$$
b(n)=\left(\begin{array}{c}
k-1+2 / q \\
k
\end{array}\right)\left(\frac{1}{2 m !}\right)^{k}
$$

\footnotetext{
${ }^{2}$ This claim is based on the fact that in [3] only a subset of the diagrams are taken into account: the result is therefore something like an upper bound on a lower bound, hence not very meaningful.
} 
for those $n$ that are of the form $n=q k+1$, as usual. For the $\phi^{4}$ theory this result coincides with that given by [2]. Asymptotically, we have

$$
a(n) \sim-i n ! n^{3-2 / q}\left(\frac{\lambda_{m}}{m_{H}^{2}}\right)^{(n-1) / q}\left(\frac{m_{H}^{2} q^{-1+2 / q}}{\Gamma(2 / q)}\right),
$$

so that, in contrast with the previous cases, the power of $n$ in the asymptotic limit does depend somewhat on $m$. Also note that the convergence limit, which was given, in the algebraic case of diagram counting, by a branch point in the generating function, is now actually a pole. Another asymptotic technique is useful here: under the assumption that, for large $n, b(n)$ behaves as $c_{1} n^{c_{2}} c_{3}^{n}$ with some constants $c_{1,2,3}$, the form of $y(x)$ close to such a pole must be $c_{0} /\left(1-c_{3} x\right)^{c_{2}+1}$, with some $c_{0}$. By inserting this form and zeroing the dominant divergence (coming from the $y^{\prime \prime}$ and the $y^{p}$ ) we can glean the information that

$$
c_{2}=-1+2 / q \quad, \quad q^{2} c_{0}^{q}=2 m ! .
$$

This is of course in agreement with Eq. (36), but this method can also be used to get information from differential equations that we cannot solve. Unfortunately, the crucial constant $c_{3}$ is not determined by such an approach.

\subsection{The mixed theory}

We now again turn to the Minimal Standard Model case of mixed $\phi^{4}$ and $\phi^{3}$ interactions. We have, for $n>1$ :

$$
\begin{aligned}
a(n)= & -i \lambda_{4} \frac{n !}{6 m_{H}^{6}} \sum_{n_{1,2,3} \geq 1} \frac{i a\left(n_{1}\right)}{n_{1} !\left(n_{1}^{2}-1\right)} \frac{i a\left(n_{2}\right)}{n_{2} !\left(n_{2}^{2}-1\right)} \frac{i a\left(n_{3}\right)}{n_{3} !\left(n_{3}^{2}-1\right)} \delta_{n_{1}+n_{2}+n_{3}, n} \\
& -i \lambda_{3} \frac{n !}{2 m_{H}^{4}} \sum_{n_{1,2} \geq 1} \frac{i a\left(n_{1}\right)}{n_{1} !\left(n_{1}^{2}-1\right)} \frac{i a\left(n_{2}\right)}{n_{2} !\left(n_{2}^{2}-1\right)} \delta_{n_{1}+n_{2}, n}
\end{aligned}
$$

and, with

$$
a(n)=-i m_{H}^{2} n !\left(n^{2}-1\right) b(n)\left(\frac{\lambda_{4}}{m_{H}^{2}}\right)^{(n-1) / 2}
$$

we find for the generating function the equation

$$
x^{2} \frac{d^{2}}{d x^{2}} y(x)+x \frac{d}{d x} y(x)-\frac{1}{6} y(x)^{3}-\frac{\mu}{2} y(x)^{2}-y(x)=0 .
$$

By the same method as above we can also solve this equation exactly, to find the surprisingly simple result

$$
\begin{aligned}
y(x) & =\frac{x}{\left(1-a_{+} x\right)\left(1-a_{-} x\right)}, \\
a_{ \pm} & =\frac{1}{12}(\mu \pm \sqrt{3}) .
\end{aligned}
$$


The resulting, exact form for the $b(n)$ is

$$
b(n)=(12)^{-n+1 / 2}\left((\mu+\sqrt{3})^{n}-(\mu-\sqrt{3})^{n}\right) .
$$

Again a special role for the value $\mu= \pm \sqrt{3}$ is evident, since in that case one of the two singularities in the generating function disappears. The asymptotic form of $a(n)$, for $\mu>0$, is

$$
a(n) \sim-i n ! n^{2}\left(\frac{\lambda_{3}(\mu+\sqrt{3})}{12 m_{H}^{2} \mu}\right)^{n}\left(\frac{12 m_{H}^{6}}{\lambda_{4}}\right)^{1 / 2}
$$

The nice and compact result (44) allows a fast determination of an absolute upper bound on the amplitude, both for the case of $\phi^{4}$ theory as in [2], and for that of broken symmetry.

A nice illustration of the connection between the unbroken and broken formulations of the theory is given by the following excercise. By making the replacement $y(x) \rightarrow z(x)=y(x)+\sqrt{3}$ in Eq. (42) we can remove the $y^{2}$ term to get

$$
x^{2} \frac{d^{2}}{d x^{2}} z(x)+x \frac{d}{d x} z(x)-\frac{1}{6} z(x)^{3}+\left(\frac{\mu^{2}}{2}-1\right) z(x)+\mu-\frac{\mu^{3}}{3}=0 .
$$

Putting $\mu=\sqrt{3}$ for simplicity, and using the expansion $z(x)=\sum_{n>0} c(n) x^{n}$ we get the recursion relation

$$
\left(n^{2}+\frac{1}{2}\right) c(n)=\frac{1}{6} \sum_{n_{1,2,3} \geq 0} c\left(n_{1}\right) c\left(n_{2}\right) c\left(n_{3}\right) \delta_{n_{1}+n_{2}+n_{3}, n} ;
$$

apart from the value $c(0)=\sqrt{3}$, the $c(n)$ are of course identical to the $b(n)$. Note, however, that the relation (47) is formally that of a pure $\phi^{4}$ theory, but with a mass term $+m_{H}^{2} / 2$ instead of $-m_{H}^{2}$ in the propagator, and a non-zero value $a(0)$ assigned to the amplitude for the decay of a single scalar into the vacuum. This is of course nothing but a reflection of the fact that the effective actions of the theory in the unbroken and broken formulations are identical, provided the field is shifted by its vacuum expectation value.

\section{A lower bound on the amplitude}

In addition to an upper bound on the cross section, it is also advisable to have a lower bound. A physically interesting result has to be of the form of either an upper bound that does not violate unitarity, or a lower bound that does: the opposite cases cannot be said to teach us anything. An estimate of a lower bound can be obtained along the same lines as in the previous section: namely, instead of finding a lower limit on the invariant masses (in the previous section given by the zero-momentum approximation) we now look for an upper limit. 
A rather trivial upper limit is given by assuming that all momentum transfers have to be smaller than $s$, the total invariant mass squared. This leads to the following recursion relation for $a(n)$ :

$$
\begin{aligned}
a(n)= & -i \lambda_{4} \frac{n !}{6 u^{3}} \sum_{n_{1,2,3} \geq 1} \frac{i a\left(n_{1}\right)}{n_{1} !} \frac{i a\left(n_{2}\right)}{n_{2} !} \frac{i a\left(n_{3}\right)}{n_{3} !} \delta_{n_{1}+n_{2}+n_{3}, n} \\
& -i \lambda_{3} \frac{n !}{2 u^{2}} \sum_{n_{1,2,3} \geq 1} \frac{i a\left(n_{1}\right)}{n_{1} !} \frac{i a\left(n_{2}\right)}{n_{2} !} \delta_{n_{1}+n_{2}, n},
\end{aligned}
$$

where $u=s-m_{H}^{2}$. Using the approach sketched in section 2 we find for the asymptotic behaviour

$$
\begin{aligned}
a(n) & \sim-i u \frac{1}{n^{3 / 2} x_{0}^{n}} \sqrt{\frac{x_{0}}{2 \pi \sqrt{2+\nu^{2}}}}\left(\frac{\lambda_{4}}{u}\right)^{(n-1) / 2}, \\
x_{0} & =\frac{1}{3}\left(-3 \nu+\nu^{3}+\left(2+\nu^{2}\right)^{3 / 2}\right) \\
\nu & =\mu m_{H} / \sqrt{u} .
\end{aligned}
$$

Although this is a valid lower bound, we see that in the limit $n \rightarrow \infty$, with $\sqrt{s} / n$ fixed, this amplitude does not go as $n$ ! but is in fact exponentially suppressed.

A better lower bound, however, can be obtained by relating the upper limit of the invariant masses to the Higgs mass $m_{H}$ rather than to $s$ : in that way the amplitude may still go like $n$ ! in the above large- $n$ limit. We propose the following. Let $\Delta$ be a number such that for any two Higgs momenta $p_{i}^{\mu}, p_{j}^{\mu}$ we have

$$
p_{i} \cdot p_{j} \leq m_{H}^{2}(1+\Delta) .
$$

We can then establish the following bound for the denominator of any of the Higgs propagators:

$$
\left(p_{1}+p_{2}+\cdots+p_{k}\right)^{2}-m_{H}^{2}<m_{H}^{2}(1+\Delta)\left(k^{2}-1\right) ;
$$

using this estimate, we find a lower bound on the propagators that scales with $m_{H}^{2}$ rather than with $s$. The recursion for the $a(n)$ becomes

$$
\begin{aligned}
a(n)= & -i \lambda_{4} \frac{n !}{6 m_{H}^{6} \omega^{3}} \sum_{n_{1,2,3} \geq 1} \frac{i a\left(n_{1}\right)}{n_{1} !\left(n_{1}^{2}-1\right)} \frac{i a\left(n_{2}\right)}{n_{2} !\left(n_{2}^{2}-1\right)} \frac{i a\left(n_{3}\right)}{n_{3} !\left(n_{3}^{2}-1\right)} \delta_{n_{1}+n_{2}+n_{3}, n} \\
& -i \lambda_{3} \frac{n !}{2 m_{H}^{4} \omega^{2}} \sum_{n_{1,2} \geq 1} \frac{i a\left(n_{1}\right)}{n_{1} !\left(n_{1}^{2}-1\right)} \frac{i a\left(n_{2}\right)}{n_{2} !\left(n_{2}^{2}-1\right)} \delta_{n_{1}+n_{2}, n}
\end{aligned}
$$

with $\omega=1+\Delta$. The Ansatz

$$
a(n)=-i m_{H}^{2} \omega n !\left(n^{2}-1\right) b(n)\left(\frac{\lambda_{4}}{m_{H}^{2} \omega}\right)^{(n-1) / 2}
$$


for which it is easily checked that $b(1)$ should again be 1 , leads to precisely the recursion relation of the zero-momentum case, but now with $\mu$ replaced by $\hat{\mu}=\mu / \sqrt{\omega}$. The solution is therefore again of the same form:

$$
b(n)=(12)^{-n+1 / 2}\left((\hat{\mu}+\sqrt{3})^{n}-(\hat{\mu}-\sqrt{3})^{n}\right) .
$$

It should be noted that the simplicity of our solution depends sensitively on the choice we made for the upper bound on the invariant mass. The crucial point is that the lefthand side of Eq. (34) contains a factor $n-1$ owing to which the differential equation (42) has no explicit inhomogeneous term. On the other hand, the even better bound $m_{H}^{2}(k-1)(k \omega+1)$ on the invariant masses does not lead to a generating function with the simple two-pole form of Eq. (43).

Before finishing we have to discuss the rôle played by $\Delta$. For given $s / m_{H}^{2}$ and $n$ the condition (50) is by no means trivial. There are two critical values $\Delta_{0}$ and $\Delta_{1}$ for $\Delta$ :

$$
\begin{aligned}
& \Delta_{0}=\left(\frac{s}{m_{H}^{2}}-n^{2}\right) /\left(n^{2}-n\right) \\
& \Delta_{1}=\frac{1}{2}\left(\frac{\sqrt{s}}{m_{H}}-n\right)^{2}+2\left(\frac{\sqrt{s}}{m_{H}}-n\right) .
\end{aligned}
$$

If $\Delta \leq \Delta_{0}$ no point in phase-space will satisfy the condition (50), and if $\Delta \geq \Delta_{1}$ all points in phase-space will satisfy it. For intermediate values of $\Delta$ a part of phase space will satisfy the condition, and our lower bound on the amplitude will only be rigorous in that part. This is no problem in principle, since by restricting ourselves to a part of phase-space the lower bound on the cross section is not invalidated. It is clear that as regards the amplitude we would like to have $\Delta$ as small as possible, but on the other hand for too small values the phase-space will be stronlgy suppressed. The balance between these two requirements will be discussed in [4].

\section{Acknowledgements}

One of us (RK) would like to thank the NIKHEF-H, Amsterdam, the Netherlands, for its kind hospitality while part of this work was carried out. EA and CP are partially supported by the EEC Program: SC1-CT91-0729.

\section{References}

[1] A. Ringwald, Nucl. Phys. B330 (1990) 1;

O. Espinosa, Cal-Tech preprint CALT-68-1586 (1989).

[2] M.B. Voloshin, Minnesota preprint TPI-MINN-92/1-T. 
[3] H. Goldberg, Phys. Lett. B246 (1990) 445.

[4] E.N. Argyres, R. Kleiss and C. Papadopoulos, in progress.

[5] N.I. Fuss, Nova Acta Academice Scientiarum Petropolitance 9 (1791) 445.

[6] J.-L. de la Grange, Mém. de l'Acad. de Berlin IV (1770).

[7] E..J. Whittaker and G.N. Watson, A Course of Modern Analysis (Cambridge University Press, 1952).

[8] H.J.M. Kuijf, Ph.D. thesis, Leiden University, 1991.

[9] H. Goldberg and M.T. Vaughn, Phys. Rev. Lett. 66 (1991) 1267.

[10] J.M. Cornwall and G. Tiktopoulos, Phys. Rev. D45 (1992) 2105. 